\title{
Neoadjuvant chemoradiation versus perioperative chemotherapy followed by surgery in resectable adenocarcinomas of the esophagogastric junction: A retrospective single center analysis
}

\author{
BJÖRN SCHULZE ${ }^{1 *}$, DOMINIK BERGIS ${ }^{2 *}$, PANAGIOTIS BALERMPAS ${ }^{1}$, JÖRG TROJAN $^{2}$, \\ GUIDO WOESTE ${ }^{3}$, WOLF OTTO BECHSTEIN ${ }^{3}$, CLAUS RÖDEL $^{1}$ and CHRISTIAN WEISS ${ }^{1}$ \\ Departments of ${ }^{1}$ Radiation Oncology, ${ }^{2}$ Gastroenterology and ${ }^{3}$ General, Visceral and Transplantation Surgery, \\ Johann Wolfgang Goethe University Hospital, Frankfurt 60590, Germany
}

Received March 24, 2013; Accepted October 10, 2013

DOI: $10.3892 /$ ol.2013.1709

\begin{abstract}
The current study presents a retrospective comparison, performed at a single academic center, of preoperative chemoradiation (CRT) and perioperative chemotherapy (CT) in addition to surgery in locally advanced but resectable adenocarcinoma of the esophagogastric junction (AEG). A total of 29 consecutive patients with locally advanced AEGs were retrospectively analyzed. Treatment consisted of preoperative CRT (mean dose, $45.0 \mathrm{~Gy}$ ) plus two cycles of CT with cisplatin and 5-FU or perioperative CT with epirubicin, cisplatin and capecitabine (three cycles preoperatively and postoperatively). Within four to six weeks following preoperative treatment, surgical therapy was performed. Median overall survival was 21.0 months in the perioperative CT group versus 41.7 months in the CRT group $[\mathrm{P}=0.36$; hazard ratio (HR), $1.50 ; 95 \%$ confidence interval (CI), 0.58-3.84]. Three-year survival rates were 55 and $38 \%$, respectively, in favor of the CRT group, and progression-free survival was 20.0 months in the CT group compared with 24.1 months in the CRT group $(\mathrm{P}=0.71$; HR, 1.19; 95\% CI, 0.46-3.05). The total number of major surgical complications was almost equal in the two groups. Margin-free resections were achieved in all patients of the CRT group, but only $76.9 \%$ of the CT group $(\mathrm{P}=0.05)$. In addition, significantly higher $\mathrm{R} 0$ resection rates and an increased number of pathological complete remissions were demonstrated in the CRT group compared with those of the $\mathrm{CT}$ group. These results appear
\end{abstract}

Correspondence to: Dr Björn Schulze, Department of Radiation Oncology, Johann Wolfgang Goethe University Hospital, Theodor Stern Kai 7, Frankfurt 60590, Germany

E-mail: bjoern.schulze@kgu.de

${ }^{*}$ Contributed equally

Key words: adenocarcinoma, esophagogastric junction, surgery, neoadjuvant chemoradiation, perioperative chemotherapy, multimodality treatment to indicate a trend for improved progression-free and overall survival for the CRT group. As postoperative morbidity and mortality rates were similar in the two groups, the results support the use of CRT for patients with advanced AEG tumors.

\section{Introduction}

The incidence of adenocarcinomas of the distal esophagus and esophagogastric junction (AEG) is rising notably in Western populations with $>480,000$ new cases diagnosed annually, accounting for 400,000 mortalities per year $(1,2)$. Despite adequate preoperative staging and improvements in perioperative treatment, the overall prognosis remains poor, with a five-year-survival rate of $\sim 40 \%$. In addition, $<30 \%$ of patients exhibit potentially operable tumors and the majority of patients already have locally advanced tumor stages with involvement of locoregional lymph nodes on presentation (3). For patients undergoing surgery following neoadjuvant therapy [chemoradiotherapy (CRT) or chemotherapy (CT) alone], three-year survival rates vary between 22 and 55\% (4-11). To improve long-term survival rates, multimodal treatment strategies, including preoperative CT and neoadjuvant CRT, for locally advanced AEGs have been considered and investigated widely. In an updated meta-analysis, Sjoquist et al summarized the results of multimodal treatment strategies indicating a trend in favor of neoadjuvant CRT (12). In addition, a current phase III trial from the Netherlands has confirmed the feasibility and superiority of a neoadjuvant $\mathrm{CR}$ regimen compared with surgery alone (10). However, a standard of care for patients with AEG tumors has not yet been defined. Recently, the EORTC expert panel voted in favor of preoperative CRT for AEG I and II tumors and recommended perioperative CT for AEG III tumors (13).

The current study presents a retrospective analysis of a single center experience with preoperative CRT or perioperative CT in addition to surgery in locally advanced but resectable AEG. The aim of the study was to identify the advantages and potential disadvantages of the two treatment regimens. Patients who were treated either with perioperative CT or neoadjuvant CRT between the years 2006 and 2012 at 
the Johann Wolfgang Goethe University Hospital (Frankfurt, Germany) were included in the analysis. Major surgical and non-surgical complications were evaluated and a Kaplan-Meier survival analysis was performed to compare the overall and progression-free survival-estimates between the two groups.

\section{Patients and methods}

Patients and treatment. A retrospective analysis was performed of patients with advanced but resectable AEG, treated in neoadjuvant intention with neoadjuvant CRT or perioperative CT between January 2006 and October 2012. Patients were allocated to the two treatment regimens almost equally by the consensus decision of a multidisciplinary tumor board of the Johann Wolfgang Goethe University Hospital. In total, 29 patients were identified who received identical CRT or CT schedules. Patients with different treatment regimens or who were participants in clinical trials were excluded from the analysis to achieve a homogeneous study population. The study was approved by the ethics committee of the Johann Wolfgang Goethe University Hospital. Written informed consent was obtained from the patients.

Preoperative staging. Initial staging included endoscopy of the upper gastrointestinal tract with multiple biopsies, computed tomography scan of the thorax and abdomen and an endoscopic ultrasound. Other diagnostics, including positron emission tomography/computed tomography or diagnostic laparotomy, were optional. Physical examination and laboratory testing were routinely performed in all patients.

CRT group. The CRT group included 16 patients. Radiation therapy planning was based on three-dimensional computed tomography scans of the chest and upper abdomen with a resolution of $3-\mathrm{mm}$ slice reconstructions. The planning target volume was delineated as the macroscopic gross tumor volume plus the safety margins of $15 \mathrm{~mm}$ in the circumferential, $30 \mathrm{~mm}$ in the oral and $50 \mathrm{~mm}$ in the aboral extension. Patients received a median cumulative dose of 45.0 Gy (range, 45.0-50.4 Gy) in single fractions of $1.8 \mathrm{~Gy} /$ day. In addition, patients received two cycles of cisplatin and 5-Fluorouracil (5-FU) in the first and fifth week of radiotherapy. Cisplatin was administered at a dose of $20 \mathrm{mg} / \mathrm{m}^{2}$ from day one to five of each cycle. 5-FU was administered at a dose of $600 \mathrm{mg} / \mathrm{m}^{2}$ as a continuous infusion from day one to five of each cycle.

CT group. The CT group included 13 patients who received a maximum of six three-week cycles of epirubicin, cisplatin and capecitabine (three cycles preoperatively and postoperatively). On day one of every three-week cycle, $50 \mathrm{mg} / \mathrm{m}^{2}$ epirubicin was administered to each patient, followed by the administration of $60 \mathrm{mg} / \mathrm{m}^{2}$ cisplatin. Between days one and $14,1,000 \mathrm{mg} / \mathrm{m}^{2}$ capecitabine was orally administered twice daily. Following surgical resection, patients underwent adjuvant treatment with the same CT regimen.

Surgery and follow-up. Surgery in the two groups was performed between four and six weeks following preoperative treatment. Extended gastrectomy and distal esophagectomy, with Roux-en-Y esophagojejunostomy and two-field D2-lymph node dissection, was performed in patients with AEG II/III. Transthoracic esophagectomy and proximal gastrectomy with en bloc removal of the esophagus and adjacent lymph nodes were performed in patients with AEG I. Patients were first seen for physical examination six to eight weeks following the termination of therapy. Technical examination (esophagogastroduodenoscopy or computed tomography) was performed at the discretion of the attending physician. For follow-up, patients were observed every three months in the first year and every six months in the following years. Medical reports and information from the attending physicians were also taken into account for analysis.

Statistical analysis. Data were analyzed and compiled using BiAS software for Windows (version 9.11; Epsilon-Verlag, Darmstadt, Germany), SPSS version 20 (SPSS, Inc., Chicago, IL, USA) and GraphPad Prism 5 for Windows (version 5; GraphPad Software Inc., La Jolla, CA, USA). P<0.05 was considered to indicate a statistically significant difference. Follow-up time was defined as the time between initiation of preoperative therapy and mortality or final contact. The primary endpoint of the study was overall survival (OS), calculated between initiation of preoperative therapy and mortality. Progression-free survival (PFS) was calculated between the initiation of neoadjuvant treatment and reported initial reaction (defined as locoregional relapse or distant metastases) or mortality.

Acute hematological side effects were recorded according to Common Toxicity Criteria, version 3.0 (http://ctep.cancer. gov/protocolDevelopment/electronic_applications/ctc.htm).

For TNM staging, the current TNM classification at diagnosis was used respecting the 2009 revision of TNM classification for esophageal cancer (www.uicc.org).

\section{Results}

Patient follow-up. Median follow-up was 25.5 months (range, 6.0-73.3 months) in the CRT group versus 22.0 months in the CT group (range, 5.3-64.7 months). Patients and tumor characteristics are shown in Table I.

Acute side effects and feasibility of perioperative and preoperative therapy. In the two groups, no acute non-hematological adverse events $\geq$ grade 3 , leading to treatment modifications, were recorded. Acute hematological toxicity grade 3 or 4 was reported more frequently in the CRT group [eight of the 16 patients (50\%)] compared with the CT group [two of the 13 patients $(15 \%)$ ] $(\mathrm{P}=0.02)$. Therefore, a dose reduction of $\mathrm{CT}$ was necessary in $50 \%$ of CRT patients and $15 \%$ of CT patients. In 15 of the 16 patients (94\%) in the CRT group and 12 of the 13 patients $(92 \%)$ in the CT group, all scheduled preoperative CT cycles were able to be administered. In the CT group, eight of the 13 patients (62\%) were unable to receive adjuvant CT due to prolonged hematological toxicity or deterioration of general condition (Table II).

Major surgical and non-surgical postoperative complications and associated mortality. Postoperative pulmonary and pleural complications, including pneumonia, pneumothorax, 
Table I. Patient and tumor characteristics.

\begin{tabular}{|c|c|c|}
\hline Characteristics & Chemoradiotherapy & Chemotherapy \\
\hline Patients, $\mathrm{n}$ & $16(100.0)$ & $13(100.0)$ \\
\hline \multicolumn{3}{|l|}{ Gender } \\
\hline Male & $13(81.0)$ & $12(92.0)$ \\
\hline Female & $3(19.0)$ & $1(8.0)$ \\
\hline \multicolumn{3}{|l|}{ Age, years } \\
\hline Median (range) & $63.8(36.4-77.6)$ & $58.8(29.1-79.1)$ \\
\hline$<50$ & $2(13.0)$ & $4(31.0)$ \\
\hline $51-60$ & $4(25.0)$ & $3(23.0)$ \\
\hline $61-70$ & $5(31.0)$ & $2(15.0)$ \\
\hline$>70$ & $5(31.0)$ & $4(31.0)$ \\
\hline \multicolumn{3}{|l|}{ Tumor site ${ }^{\mathrm{a}}$} \\
\hline AEG I & $11(69.0)$ & $8(62.0)$ \\
\hline AEG II & $3(19.0)$ & $3(23.0)$ \\
\hline AEG III & $2(12.0)$ & $2(15.0)$ \\
\hline \multicolumn{3}{|c|}{ Preoperative T stage } \\
\hline 2 & $2(12.5)$ & $4(31.0)$ \\
\hline 3 & $12(75.0)$ & $9(69.0)$ \\
\hline 4 & $2(12.5)$ & $0(0.0)$ \\
\hline \multicolumn{3}{|c|}{ Preoperative $\mathrm{N}$ stage } \\
\hline 0 & $3(19.0)$ & $4(31.0)$ \\
\hline+ & $13(81.0)$ & $9(69.0)$ \\
\hline
\end{tabular}

${ }^{a}$ AEG allocation determined by the anatomical localization of the tumor site according to Siewert's classification (14). Values are presented as $\mathrm{n},(\%)$, unless specified otherwise. AEG, adenocarcinoma of the esophagogastric junction.

Table II. Acute toxicity, treatment characteristics and comorbidity.

\begin{tabular}{lcc}
\hline Treatment & Chemoradiotherapy & Chemotherapy \\
\hline Patients & $16(100)$ & $13(100)$ \\
Acute toxicity & $0(0)$ & $0(0)$ \\
Non-hematological & $8(50)^{\mathrm{a}}$ & $2(15)^{\mathrm{a}}$ \\
Hematological (CTC grade 3/4) & $45.0(45.0-66.6)^{\mathrm{b}}$ & $\mathrm{NA}$ \\
Cumulative dose of irradiation, Gy (range) & $15(94)$ \\
Preoperative Chemotherapy & $8(50)$ & $12(92)$ \\
All scheduled cycles of chemotherapy & $\mathrm{NA}(15)$ \\
Dose reduction of chemotherapy during preoperative treatment & $5(38)$ \\
Postoperative Chemotherapy & $\mathrm{NA}$ \\
Receiving postoperative chemotherapy & \\
\hline
\end{tabular}

${ }^{\mathrm{a}} \mathrm{P}=0.02$ (Fisher's exact test); ${ }^{\mathrm{b}} 1.8 \mathrm{~Gy} /$ fraction. Values are presented as $\mathrm{n},(\%)$, unless specified otherwise. CTC, Common Terminology Criteria for Adverse Events of the National Cancer Institute (version 3.0); NA, not applicable.

relevant pleural-effusion and pleural empyema, occurred more frequently in the CRT group than in the CT group (44 vs. 8\%, respectively; $\mathrm{P}=0.04)$. One patient in each group succumbed to their condition during hospitalization due to septic complications following anastomotic leakage (Table III). The two groups did not differ significantly in the number of major surgical complications (CRT group, 69\% vs. CT group, 77\%; $\mathrm{P}=0.63$; Table IV).

Pathological complete remission and rate of $\mathrm{R} 0$ resection. Pathological complete remission (pCR) of the tumors was observed in three patients in the CT group (CT group, 19\% 
Table III. Surgical complications and mortality.

\begin{tabular}{|c|c|c|c|}
\hline Complication & Chemoradiotherapy, n (\%) & Chemotherapy, n (\%) & P-value \\
\hline Patients & $11(69)$ & $10(77)$ & NS \\
\hline \multicolumn{4}{|l|}{ Type of major surgical complication } \\
\hline Anastomotic leakage & $4(25)$ & $4(31)$ & NS \\
\hline Mediastinitis/sepsis & $1(6)$ & $1(8)$ & NS \\
\hline Implantation of esophageal stent & $2(13)$ & $2(15)$ & NS \\
\hline Pulmonary complications & $7(44)$ & $1(8)$ & 0.04 \\
\hline Secondary surgery & $3(19)$ & $1(8)$ & NS \\
\hline Necrosis of intrathoracic gastric tube/neoesophagus & $1(6)$ & $0(0)$ & NS \\
\hline Lymphatic fistula & $1(6)$ & $0(0)$ & NS \\
\hline Other & $5(31)$ & $3(23)$ & NS \\
\hline Complication-associated mortality & $1(6)$ & $1(8)$ & NS \\
\hline
\end{tabular}

aDetermined by Fisher's exact test. NS, not significant.

Table IV. Surgical outcome and survival data.

\begin{tabular}{|c|c|c|c|}
\hline Outcome & Chemoradiotherapy & Chemotherapy & P-value \\
\hline $\mathrm{R} 0$ resection $^{\mathrm{b}}$ & $16(100)$ & $10(77)$ & 0.05 \\
\hline $\mathrm{pCR}^{\mathrm{c}}$ & $3(19)$ & $0(0)$ & 0.23 \\
\hline Median OS, months & 41.7 & 21.0 & 0.36 \\
\hline 3-year OS rate, $\%$ & 55.0 & 38.0 & NS \\
\hline Median PFS, months & 24.1 & 20.0 & 0.71 \\
\hline \multicolumn{4}{|l|}{ Pattern of recurrence } \\
\hline Locoregional & $2(13)$ & $4(31)$ & NS \\
\hline Distant & $7(44)$ & $4(31)$ & NS \\
\hline Locoregional and distant & $1(6)$ & $1(8)$ & NS \\
\hline
\end{tabular}

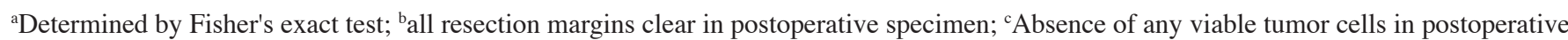
specimen. Values are presented as n, (\%), unless specified otherwise. pCR, pathological complete regression; OS, overall survival; PFS, progression-free survival; NS, not significant.

vs. CRT group, $0 \% ; \mathrm{P}=0.11)$. In addition, the $\mathrm{R} 0$ resection rate (complete tumor-free resection margins) was significantly higher in the CRT group compared with the CT group (100 vs. 77\%, respectively; $\mathrm{P}=0.05$; Table IV).

OS and PFS. Median OS time was 21.0 months in the CT group versus 41.7 months in the CRT group $[\mathrm{P}=0.36$; hazard ratio (HR), 1.50; 95\% confidence interval (CI), 0.58-3.84]. Three-year survival rates were $38 \%$ in the CT group and $55 \%$ in the CRT group. Median PFS time was 20.0 months in the CT group compared with 24.1 months in the CRT group ( $\mathrm{P}=0.71$; HR, 1.19; 95\% CI, 0.46-3.05) (Table IV; Figs. 1 and 2).

Patterns of recurrence and secondary malignancies detected during the follow-up period. The rate of local and/or distant recurrence during the follow-up period was $56 \%$ in the CRT and $69 \%$ in the CT group (Table IV). In the CRT group, three patients were diagnosed with secondary malignancies, including non-small cell lung cancer, colon carcinoma and cholangiocarcinoma. At least one patient in the CRT group succumbed to a secondary malignancy and not to the AEG tumor. In the other two cases, the cause of mortality was not clearly identifiable.

\section{Discussion}

Although modern perioperative treatment regimens have shown improved outcomes for patients with advanced AEG tumors, there remains a lack of profound data to define the most effective treatment approach. The current study compared the outcome of a homogeneous patient population treated with preoperative CRT or perioperative CT at a single center. An updated meta-analysis in 2011 analyzing the administration of neoadjuvant CT or CRT in addition to surgery was unable to determine a clear advantage between the treatment regimens, although, a proportionately larger 


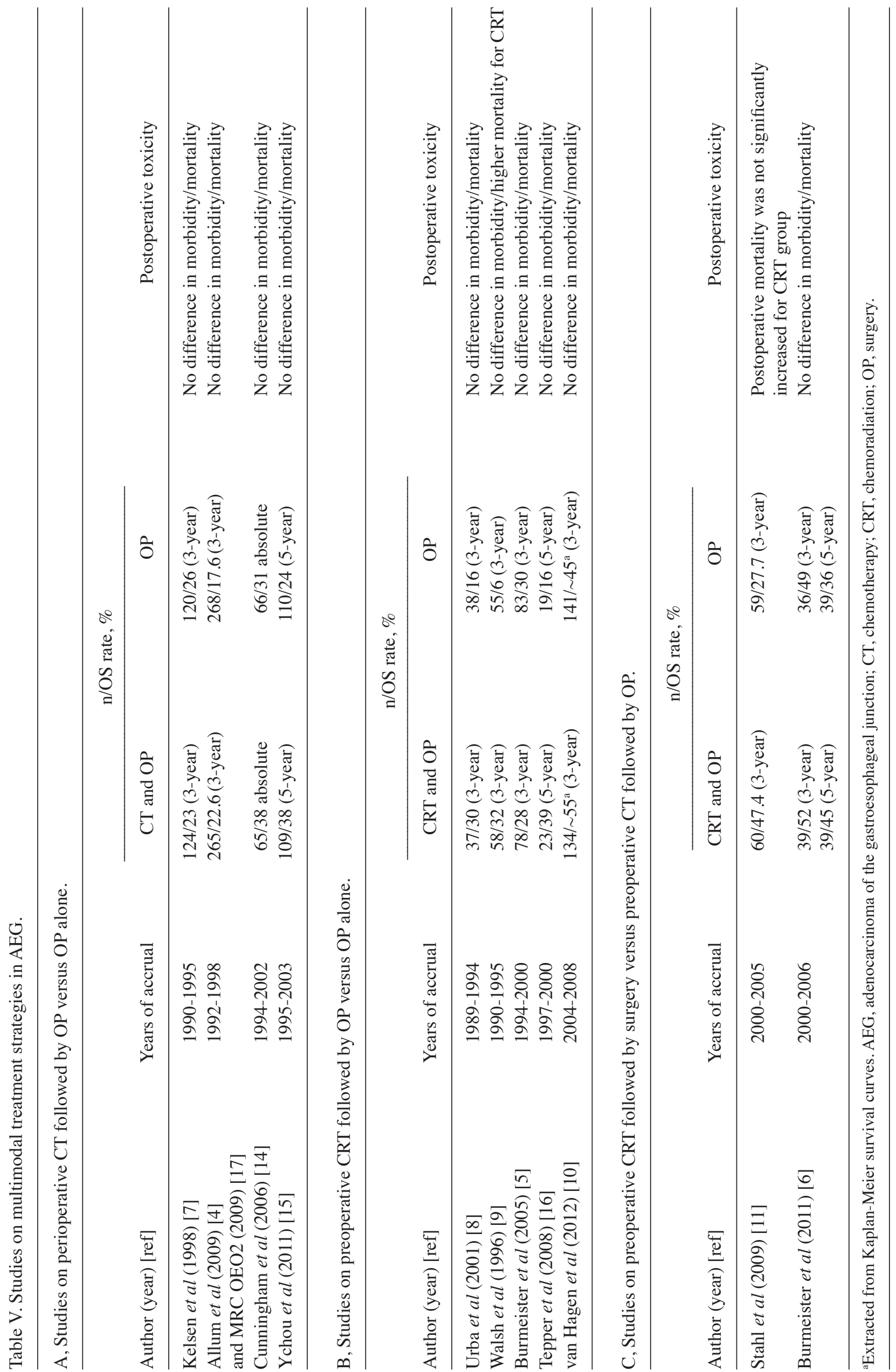




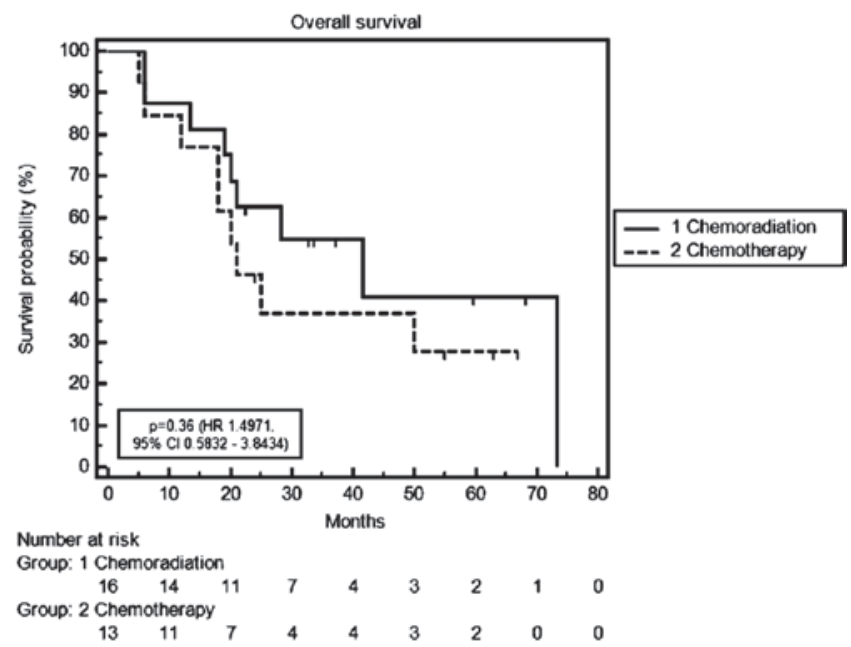

Figure 1. Overall survival probability for the two groups (neoadjuvant chemoradiation and perioperative chemotherapy) figured as Kaplan-Meier survival curves. There was no significant difference among the two groups (log rank test, $\mathrm{P}=0.36$ ). HR, hazard ratio; $\mathrm{CI}$, confidence interval.

survival benefit was observed for CRT versus CT (12) However, concern remains that this benefit is achieved at the expense of an increase in morbidity and mortality. The present study found no significant differences in overall morbidity and mortality between the two treatment arms. This is consistent with the results of a number of randomized trials that found similar overall morbidity and mortality rates for perioperative $\mathrm{CT}$ and preoperative CRT when compared with surgery alone (Table V) (4-11,15-18). However, in the current study, a significantly higher rate of pulmonary complications (44\%) was observed in the CRT compared with the CT group. Similar rates ranging between 20 and $50 \%$ have been reported by a number of authors investigating neoadjuvant CRT followed by surgery versus surgery alone. Notably, in these studies, the two groups (neoadjuvant CRT plus surgery vs. surgery alone) showed similar pulmonary morbidity rates. This indicates that pulmonary complications are not likely to be predominantly caused by the addition of radiotherapy or CT alone $(6,8-10,19)$. However, the differences demonstrated in the current study are not well explained and highlight issues of target volume definition, radiotherapy dose/fractionation and lung sparing techniques, which must be accounted for in future studies. An additional difference between the treatment groups was observed in the frequency of hematological side effects, with 50\% of the patients in the CRT group developing grade $3 / 4$ hematotoxicity compared with only $15 \%$ in the CT group. The extremely low hematotoxicity in the CT group is contradictory to an additional single center phase II study investigating a similar preoperative $\mathrm{CT}$ regimen (epirubicin/cisplatin/capecitabine) that only differed by the dose of capecitabine. In this trial, the reported grade $3 / 4$ neutropenia was $62 \%$ (20). By contrast, a recent phase III trial comparing preoperative CRT in addition to surgery with surgery alone reported considerably low rates of grade 3 and 4 hematotoxicity $(<10 \%)$ with a treatment compliance of $>90 \%$, using a new chemotherapeutic regimen consisting of carboplatin and paclitaxel, with a radiation dose of 41.1 Gy in 1.8 Gy fractions. No differences in morbidity or mortality were observed

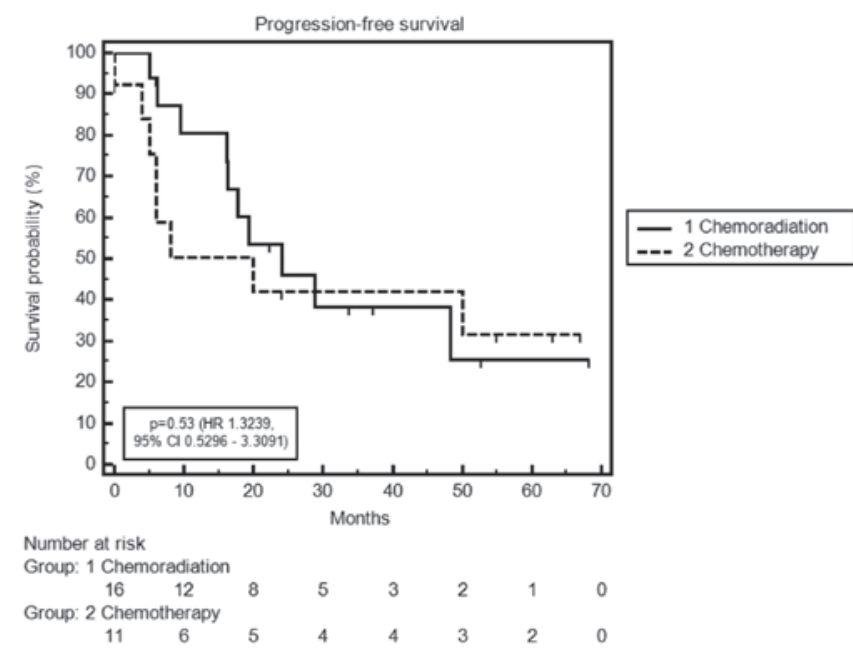

Figure 2. Progression-free survival probability for the two groups (neoadjuvant chemoradiation and perioperative chemotherapy) figured as Kaplan-Meier survival curves. There was no significant difference among the two groups ( $\log$ rank test, $\mathrm{P}=0.71$ ). $\mathrm{HR}$, hazard ratio; $\mathrm{CI}$, confidence interval.

in the two groups, however, patients in the CRT group showed significantly improved survival outcomes (10). Previously, the long-term results of the MRC OEO2 trial, which compared the additional effect of preoperative $\mathrm{CT}$ with surgery alone, showed that patients with microscopically complete resection (R0 resection) exhibited an OS rate of $42.4 \%$ compared with $18 \%$ of patients with microscopically incomplete resection (R1 resection) and $8.6 \%$ of patients with a remaining macroscopic tumor ( $\mathrm{R} 2$ resection) (4). The present series identified a significantly higher $\mathrm{R} 0$ resection rate for patients who received CRT (100\%) compared with those who received CT (77\%). Furthermore, only patients in the CRT group $(n=3)$ achieved pCR. Consecutively, a non-significant trend to a higher PFS and OS and an improved local control rate was identified in the CRT group. By contrast, preoperative CT was noted to decrease the incidence of distant metastasis by 31 versus $44 \%$ in the CRT group. Overall, these results reflect the results of the only two randomized trials that have directly compared preoperative CT with preoperative CRT. However, the two trials closed prematurely due to poor accrual. Stahl et al reported three-year OS rates of $47.4 \%$ in the CRT group and $27.7 \%$ in the CT group, with an increased number of patients in the CRT group experiencing pathological downstaging and pCR compared with the CT group (15.6 vs. $2 \%$, respectively). Notably, all patients with pCR survived (11). Similarly, Burmeister et al was unable to demonstrate a significant survival benefit with the addition of radiation therapy to preoperative CT. The authors reported a prolonged time to progression, a significantly higher $\mathrm{pCR}$ rate and a trend to an improved R0 resection rate in the CRT group. The reported OS rates at three years were 49 (CT) versus 52\% (CRT) and are consistent with the current results (6).

To determine the best multimodal treatment regimen, further studies are required. At present, an ongoing study, the international phase III TOPGEAR trial (launched in 2012), is investigating whether preoperative CRT (two cycles of ECF followed by $45 \mathrm{~Gy}$ of radiation with concurrent $5-\mathrm{FU}$ ) or preoperative CT (three cycles of ECF) alone is more effective 
in patients with resectable gastric and esophagogastric cancer. Following surgery, the two groups were scheduled to receive three additional cycles of ECF (unpublished data).

Despite the major limitations of the present small and retrospective analysis, the results confirmed the results of recent randomized trials addressing the issue of whether preoperative CT or CRT is superior for the treatment of AEG tumors. The current study demonstrated significantly higher R0 resection rates and an increased number of pCR in the CRT group. These results appear to indicate a trend for improved PFS and OS for the CRT group. As postoperative morbidity and mortality rates were similar in the two groups, the results of the current study support the use of CRT for patients with advanced AEG. Nevertheless, large trials integrating the best available treatment schedules are required to define a standard treatment approach for this increasingly common tumor entity.

\section{References}

1. Pera M: Epidemiology of esophageal cancer, especially adenocarcinoma of the esophagus and esophagogastric junction. Recent Results Cancer Res 155: 1-14, 2000.

2. Trivers KF, Sabatino SA and Stewart SL: Trends in esophageal cancer incidence by histology, United States, 1998-2003. Int J Cancer 123: 1422-1428, 2008.

3. Breaux JR, Bringaze W, Chappuis C and Cohn I Jr: Adenocarcinoma of the stomach: a review of 35 years and 1,710 cases. World J Surg 14: 580-586, 1990.

4. Allum WH, Stenning SP, Bancewicz J, Clark PI and Langley RE: Long-term results of a randomized trial of surgery with or without preoperative chemotherapy in esophageal cancer. J Clin Oncol 27: 5062-5067, 2009.

5. Burmeister BH, Smithers BM, Gebski V, et al; Trans-Tasman Radiation Oncology Group; Australasian Gastro-Intestinal Trials Group: Surgery alone versus chemoradiotherapy followed by surgery for resectable cancer of the oesophagus: a randomised controlled phase III trial. Lancet Oncol 6: 659-668, 2005.

6. Burmeister BH, Thomas JM, Burmeister EA, et al: Is concurrent radiation therapy required in patients receiving preoperative chemotherapy for adenocarcinoma of the oesophagus? A randomised phase II trial. Eur J Cancer 47: 354-360, 2011.

7. Kelsen DP, Ginsberg R, Pajak TF, et al: Chemotherapy followed by surgery compared with surgery alone for localized esophageal cancer. N Engl J Med 339: 1979-1984, 1998.

8. Urba SG, Orringer MB, Turrisi A, Iannettoni M, Forastiere A and Strawderman M: Randomized trial of preoperative chemoradiation versus surgery alone in patients with locoregional esophageal carcinoma. J Clin Oncol 19: 305-313, 2001.
9. Walsh TN, Noonan N, Hollywood D, Kelly A, Keeling N and Hennessy TP: A comparison of multimodal therapy and surgery for esophageal adenocarcinoma. N Engl J Med 335: 462-467, 1996.

10. van Hagen P, Hulshof MC, van Lanschot JJ, et al; CROSS Group: Preoperative chemoradiotherapy for esophageal or junctional cancer. N Engl J Med 366: 2074-2084, 2012.

11. Stahl M, Walz MK, Stuschke M, et al: Phase III comparison of preoperative chemotherapy compared with chemoradiotherapy in patients with locally advanced adenocarcinoma of the esophagogastric junction. J Clin Oncol 27: 851-856, 2009.

12. Sjoquist KM, Burmeister BH, Smithers BM, et al; Australasian Gastro-Intestinal Trials Group: Survival after neoadjuvant chemotherapy or chemoradiotherapy for resectable oesophageal carcinoma: an updated meta-analysis. Lancet Oncol 12: 681-692, 2011.

13. Lutz MP, Zalcberg JR, Ducreux M, et al; First St Gallen EORTC Gastrointestinal Cancer Conference 2012 Expert Panel: Highlights of the EORTC St. Gallen International Expert Consensus on the primary therapy of gastric, gastroesophageal and oesophageal cancer - differential treatment strategies for subtypes of early gastroesophageal cancer. Eur J Cancer 48: 2941-2953, 2012

14. Siewert JR, Hölscher AH, Becker K and Gössner W: Cardia cancer: attempt at a therapeutically relevant classification. Chirurg 58: 25-32, 1987 (In German).

15. Cunningham D, Allum WH, Stenning SP, et al; MAGIC Trials Participants: Perioperative chemotherapy versus surgery alone for resectable gastroesophageal cancer. N Engl J Med 355: 11-20, 2006.

16. Ychou M, Boige V, Pignon JP, et al: Perioperative chemotherapy compared with surgery alone for resectable gastroesophageal adenocarcinoma: an FNCLCC and FFCD multicenter phase III trial. J Clin Oncol 29: 1715-1721, 2011.

17. Tepper J, Krasna MJ, Niedzwiecki D, et al: Phase III trial of trimodality therapy with cisplatin, fluorouracil, radiotherapy, and surgery compared with surgery alone for esophageal cancer: CALGB 9781. J Clin Oncol 26: 1086-1092, 2008.

18. Medical Research Council Oesophageal Cancer Working Group: Surgical resection with or without preoperative chemotherapy in oesophageal cancer: a randomised controlled trial. Lancet 359: 1727-1733, 2002.

19. Leibl BJ, Vitz S, Schäfer W, Alfrink M, Gschwendtner A and Grabenbauer GG: Adenocarcinoma of the esophagogastric junction: neoadjuvant radiochemotherapy and radical surgery: early results and toxicity. Strahlenther Onkol 187: 231-237, 2011.

20. Starling N, Okines A, Cunningham D, et al: A phase II trial of preoperative chemotherapy with epirubicin, cisplatin and capecitabine for patients with localised gastro-oesophageal junctional adenocarcinoma. Br J Cancer 100: 1725-1730, 2009. 